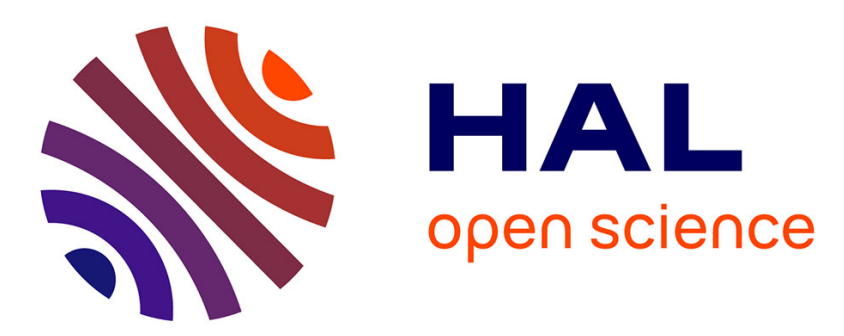

\title{
Hall-Petch Law Revisited in Terms of Collective Dislocation Dynamics
}

François Louchet, Jérôme Weiss, Thiebaud Richeton

\section{To cite this version:}

François Louchet, Jérôme Weiss, Thiebaud Richeton. Hall-Petch Law Revisited in Terms of Collective Dislocation Dynamics. Physical Review Letters, 2006, 97 (075504), 1 à 4 p. 10.1103/PhysRevLett.97.075504 . insu-00375537

\section{HAL Id: insu-00375537 https://hal-insu.archives-ouvertes.fr/insu-00375537}

Submitted on 13 Jan 2022

HAL is a multi-disciplinary open access archive for the deposit and dissemination of scientific research documents, whether they are published or not. The documents may come from teaching and research institutions in France or abroad, or from public or private research centers.
L'archive ouverte pluridisciplinaire HAL, est destinée au dépôt et à la diffusion de documents scientifiques de niveau recherche, publiés ou non, émanant des établissements d'enseignement et de recherche français ou étrangers, des laboratoires publics ou privés.

\section{(c)(1)}

Distributed under a Creative Commons Attribution| 4.0 International License 


\title{
Hall-Petch Law Revisited in Terms of Collective Dislocation Dynamics
}

\author{
François Louchet, ${ }^{*}$ Jérôme Weiss, ${ }^{\dagger}$ and Thiebaud Richeton ${ }^{*}$ \\ Laboratoire de Glaciologie et de Géophysique de l'Environnement, Université Joseph Fourier/CNRS, \\ BP96 - 38402 Saint Martin d'Hères, France
}

(Received 13 April 2006; published 15 August 2006)

\begin{abstract}
The Hall-Petch (HP) law, that accounts for the effect of grain size on the plastic yield stress of polycrystals, is revisited in terms of the collective motion of interacting dislocations. Sudden relaxation of incompatibility stresses in a grain triggers aftershocks in the neighboring ones. The HP law results from a scaling argument based on the conservation of the elastic energy during such transfers. The Hall-Petch law breakdown for nanometric sized grains is shown to stem from the loss of such a collective behavior as grains start deforming by successive motion of individual dislocations.
\end{abstract}

DOI: 10.1103/PhysRevLett.97.075504

The well-known Hall-Petch (HP) law, discovered in $1951[1,2]$, states that the grain size dependence of the macroscopic plastic yield stress of a polycrystal is given by:

$$
\sigma_{y}=\sigma_{\infty}+k d^{-1 / 2}=\sigma_{\infty}+K \mu \sqrt{b / d},
$$

where $d$ is the grain size, $k, K$, and $\sigma_{\infty}$ are constants, $\mu$ is the shear modulus, and $b$ is the Burgers vector modulus. Assuming that Eq. (1) can be extrapolated up to infinite grain sizes (which will be shown here not to be correct in most cases), $\sigma_{\infty}$ would be the yield stress of the single crystal. The typical values for pure fcc metals are $\sigma_{\infty} \approx$ $10^{-4} \mu$, and $K=k /(\mu \sqrt{b}) \cong 0.05$ to 0.5 , where the bulk modulus $E$ is related to the shear modulus $\mu$ and the Poisson's ratio $\nu$ by: $\mu=E /[2(1+\nu)]$. The value of $K$ in Hall's original paper [1] in mild steels is 0.5 , and is around 0.4 in Petch's paper for cleavage stress in both Fe and mild steels. These figures show that $\sigma_{\infty}$ is negligible in this case as compared to the grain size dependent term for grain sizes up to about several tens of microns.

Most explanations proposed so far are based on stress concentrations experienced by grain boundaries (GBs) at the head of dislocation piled-up groups ("pileups") [1,3], but suffer from the fact that such pileups are scarcely or never observed in materials that nevertheless obey the HP law, as Al for instance. Another idea [4] is based on both (i) an inverse relationship between dislocation density and grain size [5] and (ii) Taylor's relation $\sigma_{y}=\alpha \mu b \sqrt{\rho}$ between yield stress and dislocation density [6]. However, the original Taylor's relation accounts for "forest hardening," i.e., gives the stress necessary to move a dislocation through a density $\rho$ of other dislocations. It coincides with yield stress when dislocation arrays are the only obstacles to dislocation motion, which is obviously not the case for grain size hardening, and it is not always valid, more particularly when dislocation motion is controlled by Peierls-like lattice friction. Furthermore, transmission of plastic flow through GBs, which is an unavoidable feature of generalized plasticity at macroscopic plastic yield, is not explicitly considered in [4].
PACS numbers: 62.20.Fe, 61.82.Rx, 62.20. $-\mathrm{x}, 62.25 .+\mathrm{g}$

A new insight into this problem will be brought here in terms of collective dislocation dynamics. It is now agreed indeed that, at least if dislocation mobility is high, crystals deform in an intermittent manner [7,8] through strain bursts consisting of dislocation avalanches. Recent acoustic emission (AE) experiments on ice and on metallic single crystals $[7,9]$ showed that sizes of dislocation avalanches are characterized by universal scale-free (power law) distributions, suggesting a common marginally stable, critical behavior for collective dislocation dynamics.

In polycrystals, the AE experiments on ice mentioned above showed that grain boundaries act as strong obstacles to avalanches. As a result [10], a cutoff appears in size distributions that corresponds to the maximum possible area swept off by a dislocation avalanche in a grain of a given size. In addition, the size distribution of smaller avalanches has a different power law exponent than that of single crystals. This unexpected feature was interpreted in terms of aftershocks characteristic of a supercritical regime [10]: Monte Carlo simulations showed that, if the energy involved in an avalanche is too large for being relaxed in a given grain, the excess energy is redistributed to other grains as aftershocks that may relax in turn this transferred energy, and so on. This transmission of plastic flow (and not necessarily of dislocations) through GBs is reminiscent of generalized plasticity [see [11], p. 267], characteristic of the macroscopic yield (or "macroyield"), at which HP law is obeyed. These views are supported by previous findings on $\mathrm{AE}$ on ice polycrystals under creep loading: this "generalized plasticity" was characterized by (i) a fractal pattern of avalanche locations [12], i.e., a correlation length much larger than the grain size, and of the order of the sample size, and (ii) a space-time coupling between avalanches that illustrates the idea of "strain diffusion" and "percolation."

On the other hand, the HP law is no more obeyed as grain sizes go down to less than a few tens of nanometers [13]. The first theoretical attempt accounting for the HP law breakdown in such nanostructured materials relied on dimensional arguments $[14,15]$. It was proposed on the early statement by $\mathrm{Li}$ [16] that dislocations in nanosized 
grains are nucleated at GBs, travel across the grain, and annihilate on the opposite GB. This model allowed an easy derivation of the various dependencies of the flow stress versus grain size for both multilayers and polycrystals with nanosized grains (referred to as nanocrystals in the following). The same scaling arguments were used again some time later $[4,17]$, leading to more or less similar conclusions.

The first part of the present Letter aims at revisiting the HP law using both scaling arguments and the new perspectives offered by the intermittent character of plastic flow described above. As for the HP law breakdown at nanometric grain sizes, it will be discussed in the second part of the Letter as a breakdown of the intermittent flow, related to the loss of the collective behavior of dislocations.

Let us start with the case of polycrystals with mesosized grains. If Eq. (1) was valid up to infinite grain sizes, $\sigma_{\infty}$ should appear to be the yield stress of the corresponding single crystal. The $d^{1 / 2}$ term is therefore related with the effect of GBs, and accounts for the incompatibility part of the stress.

Considering a grain in a polycrystal loaded at constant imposed strain rate, the corresponding external stress $\sigma$ first increases linearly with time (elastic regime). The grain then starts deforming when $\sigma$ reaches a value $\Sigma_{o}$, which is the "true" yield stress of the corresponding single crystal with similar orientation, referred to as "friction stress" in the following. The relation between $\Sigma_{o}$ and $\sigma_{\infty}$ will be discussed further.

As neighbor grains have different orientations and slip geometries, back stresses $\delta \sigma$ (known as "incompatibility" stresses) gradually build up in the grain. The effective stress experienced by the grain interior becomes:

$$
\sigma_{\text {eff }}=\sigma-\delta \sigma .
$$

Continuing deformation of the grain at imposed strain rate requires $\sigma_{\text {eff }}=\Sigma_{o}$ that writes:

$$
\sigma=\Sigma_{0}+\delta \sigma
$$

This means that the gradual increase of incompatibility stresses $\delta \sigma$ results in a similar increase of the external stress $\sigma$, at constant $\Sigma_{o}$, until $\sigma$ reaches the macroyield stress $\sigma_{y}$, that corresponds by definition to the onset of generalized plasticity. The macroyield stress $\sigma_{y}$ thus appears as the value of $\sigma$ for which the excess energy related to incompatibility stresses can be relaxed through a transfer to neighboring grains, enhancing their plastic activity up to generalized plastic flow. As plasticity takes place through dislocation avalanches [7-10], such a transfer results from avalanches that may increase the effective stress $\sigma_{\text {eff }}$ in at least one of the neighboring grains up to a significant level above $\Sigma_{o}$, making possible further relaxations through aftershock triggerings in other grains, and so on, in some kind of "domino effect." In the same way as macroyield in a single crystal (in the absence of work hardening) corresponds to the stress for which the maximum avalanche size diverges [18], it corresponds in a polycrystal to the stress for which the correlation length of strain bursts tends to infinity, letting strain "percolate" through the material.

The maximum excess energy relaxed in a grain of diameter $d$ is the difference between the stored elastic energies corresponding to stresses $\Sigma_{o}+\delta \sigma$ and $\Sigma_{o}$, i.e.:

$$
\frac{\left(\Sigma_{o}+\delta \sigma\right)^{2}-\Sigma_{0}^{2}}{2 E} \frac{\pi d^{3}}{6}
$$

where $\pi d^{3} / 6$ is the grain volume, taken spherical for the sake of simplicity. This energy is supposed to spread on the GB surface of the order of $\pi d^{2}$. The corresponding energy density (i.e., stored elastic energy per unit volume) at the GB (supposed to have a thickness $b$ ) is

$$
W=\frac{\left(\Sigma_{o}+\delta \sigma\right)^{2}-\Sigma_{0}^{2}}{2 E} \frac{d^{3}}{6 d^{2} b}=\frac{2 \Sigma_{0} \delta \sigma+\delta \sigma^{2}}{2 E} \frac{d}{6 b}
$$

which corresponds to a local stress at the GB of the order of:

$$
\sigma_{\mathrm{loc}}=\sqrt{2 E W}=\sqrt{\left(2 \Sigma_{0} \delta \sigma+\delta \sigma^{2}\right) \frac{d}{6 b}}
$$

This quantity represents the additional stress available at the GB that may activate plasticity in the neighbor grains. Such a situation is achieved when this additional stress reaches a threshold $\Phi$, that represents the stress necessary to nucleate dislocations from the grain boundary:

$$
\sqrt{\left(2 \Sigma_{0} \delta \sigma+\delta \sigma^{2}\right) \frac{d}{6 b}}=\Phi .
$$

Solving this $2^{d}$ degree equation in $\delta \sigma$ gives:

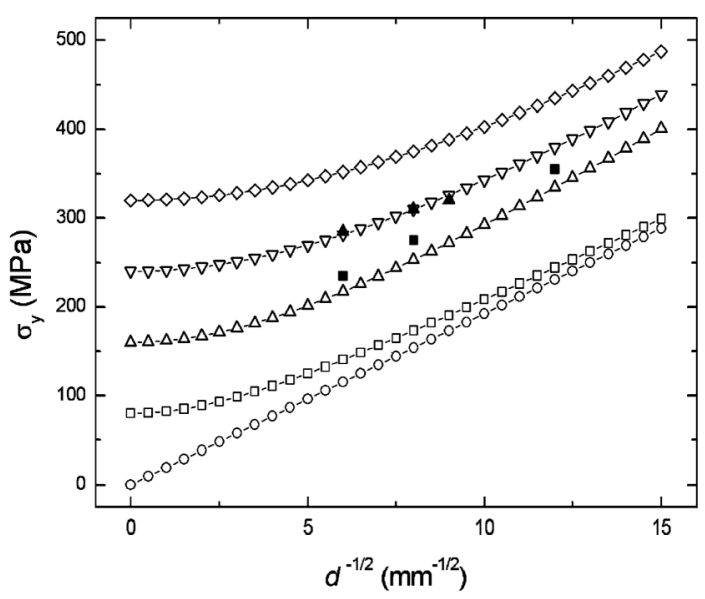

FIG. 1. Example of yield stresses $\sigma_{y}$ vs $d^{-1 / 2}$ computed from Eq. (8), in the case of iron-based alloys, taking $\mu=77 \mathrm{GPa}$, $b=0.248 \mathrm{~nm}$, and $\Phi=20 \mathrm{GPa}$. Different curves are for different values of the friction stress $\Sigma_{0}(0,80,160,240,320 \mathrm{MPa}$ from bottom to top of the figure), and are compared with experimental data for $\mathrm{Fe}-\mathrm{Si}$ alloys with two different $\mathrm{Si}$ contents, 0.025 (closed squares) and $0.29 \mathrm{wt} \%$ (closed triangles), i.e., increasing friction stress $\Sigma_{0}$ [19]. 


$$
\sigma_{y}=\Sigma_{0}+\delta \sigma=\sqrt{\Sigma_{0}^{2}+\frac{6 b}{d} \Phi^{2}},
$$

where the yield stress $\sigma_{y}$ is the value of $\sigma$ [given by Eq. (3)] when condition (7) is achieved.

Figure 1 shows an example of yield stress variations versus $d^{-1 / 2}$, computed from Eq. (8) for different values of the friction stress $\Sigma_{0}$. The value of $\Phi$ is chosen in order to fit experimental data on Fe-Si alloys [19], in which lattice friction is closely related with Si content. The best fit is for $\Phi=20 \mathrm{GPa}$. Comparing with Eq. (1), such a threshold stress corresponds to $K=0.6$, to be compared with Hall's original value $K=0.5$. It is also worth noting that $\Phi$ is of the order of the theoretical elastic limit, i.e., a fraction of the shear modulus $\mu$. This finding also agrees with experiment. Indeed, in materials in which dislocation pileups actually exist (which is a particular case of the present model), pileups of a several tens of dislocations locked on GBs are currently observed [20], leading to dislocation emission from the GB into the neighbor grain [21]. As the stress at the head of a pileup of $n$ dislocations is $n$ times larger than the applied stress, very large stresses, close to the theoretical elastic limit, can be built up allowing such dislocation emission events.

Figure 1 shows that the HP law is perfectly obeyed (as expected) in the absence of friction stress. Increasing friction stresses are responsible for a gradual deviation from HP law, more apparent at large grain sizes. At small grain sizes, i.e., when the contribution of GBs as barriers to long range extensive plasticity dominates, friction stresses still have an influence, but contributions of GBs and of friction stresses are not additive.

These findings probably explain why data obtained from hardened alloys in a limited grain size range seem to obey a HP law with an apparent parameter $k$ that decreases as friction stress increases [e.g., [19]]. This measured parameter has no direct physical meaning, and extrapolations of such data to infinite grain size should give $\sigma_{\infty}$ values that may strongly underestimate the "true" friction stress (i.e., the single crystal yield stress) $\Sigma_{o}$.

It is also worth noting that the validity of a Taylor-like relation in polycrystals cannot be considered here as a prerequisite that may be used [as it was in [4]] in the derivation of the HP law. It appears instead as a consequence of both the HP law itself and the (geometrically obvious) inverse relation between grain size and the density $\rho$ of "geometrically needed dislocations" that accommodate plastic incompatibilities

$$
\rho=\varepsilon / b d .
$$

We obtain indeed a Taylor-like relation at conventional yield from Eqs. (8) and (9), taking $\Sigma_{0}=0$ and $\varepsilon=\varepsilon_{0.2}=$ $0.2 \%$ :

$$
\sigma_{y}=\alpha \mu b \sqrt{\rho}
$$

with $\alpha=(\Phi / \mu) \sqrt{6 / \varepsilon_{0.2}}$. Yet, the possible validity of such a Taylor-like relation in a polycrystal does not mean at all that dislocation motion is controlled by forest interactions. This is probably why $\alpha(\alpha=14$ here) may significantly differ from the value expected for forest hardening ( $\alpha=$ $0.5)$. The present Taylor-like relation has nothing to do with forest hardening. It is a mere consequence of the HP law rather than a prerequisite.

The present derivation of the HP law takes into account the essential feature of generalized plasticity in a polycrystal, which is transmission of plasticity (and not necessarily of dislocations) through GBs and its "percolation" through the material.

This model is quite general, since it does not require any particular assumption as, for instance, the existence of dislocation pileups (that appears as a particular case), or any other detail about dislocation structures or interactions or deformation mechanisms. The existence of dislocations themselves should not be required. For instance, the same argument may be applied to the case where correlated dislocation motion is replaced by correlated crack openings, which may explain why the HP law is also obeyed for cleavage of polycrystals, which was actually the case reported in Petch's original paper in 1953 [2].

Let us now examine the nanometric grain size range. Decreasing the grain size has various consequences: (i) a departure from HP behavior is reported in most metals for grain sizes less than a few $\mathrm{nm}$, changing into a stress saturation or even a softening at smaller grain sizes $[22,23]$. (ii) Since the Burgers vector modulus is a constant, the strain $b / d$ provided by a single dislocation crossing a grain increases as the grain size goes down. In other words, the number of dislocations involved in a strain burst of a given size decreases, suggesting a gradual vanishing of both collective dislocation motion and avalanche amplitudes. This is confirmed by the vanishing of AE signals, i.e., of intermittent flow, for grain sizes smaller than $800 \mathrm{~nm}$ [24]. (iii) More precisely, as the number $N$ of geometrically needed dislocations in a grain is of the order of $\rho d^{2}$, it can be deduced from Eq. (9) that $N=(\varepsilon / b) d$ [which is equivalent to the above remark (ii)]. As a consequence, grains smaller than $b / \varepsilon$ (typically $100 \mathrm{~nm}$ at yield) should hardly contain dislocations. Deformation of the corresponding polycrystals should therefore proceed through direct transfer across the grain of individual dislocations from a GB where they are nucleated to the opposite one at which they annihilate, the kinetics being controlled by the nucleation process. Such a mechanism, predicted by Li [16], was confirmed experimentally [25], and reproduced by molecular dynamics simulations [17].

Let us consider strain rates large enough to neglect diffusional processes. As strain proceeds through intermittent nucleations of individual dislocations at GBs, with flight times much smaller than nucleation times, the strain rate is given by:

$$
\dot{\varepsilon}=\nu(\sigma) \frac{b}{d},
$$




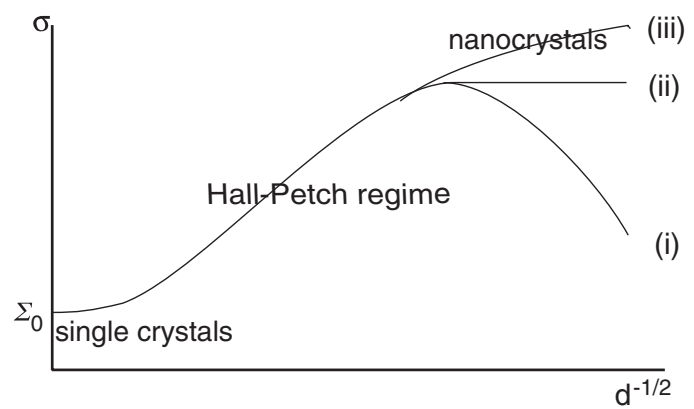

FIG. 2. Schematic variations of the macroyield stress vs $d^{-1 / 2}$ : from the single crystal limiting case $\Sigma_{o}$, the macroyield stress increases as $d^{-1 / 2}$ in the HP range. At nanoscales, the HP breakdown leads to three possible behaviors according whether dislocations are nucleated at vertices (i), at GB triple junctions (ii), or at GBs (iii).

where $b / d$ is the strain corresponding to a single dislocation nucleated at a GB and crossing the whole grain, and $\nu(\sigma)$ is the stress-dependent nucleation frequency at the GB, which includes the number of possible nucleation sites that may act in parallel.

Different grain size dependencies of the strain rate are obtained, depending on the scaling of the number of nucleation sites $[14,15]$ : the number of nucleation sites per grain scales as $d^{2}$ if nucleation takes place at GBs, but as $d$ for triple junctions (i.e., lines separating 3 grains), and is constant for vertices. Combining with Eq. (11), the strain rate turns out to be proportional to the grain size $d$ in the first case, constant in the second one, and proportional to $1 / d$ in the last one. The first case leads to hardening as grain size is reduced, as observed in Fe [26] or NiP nanocrystals, the second one to saturation of the flow stress, and the third one to softening, as reproduced by Yamakov's MD simulations [27]. These results are schematized in Fig. 2.

To conclude, the main reason for the breakdown of the HP law at nanometric grain sizes therefore appears to be that collective effects vanish, as attested by the disappearance of AE signals. Instead of involving intermittent and correlated motion of interacting dislocations, strain proceeds through uncorrelated events of individual dislocation nucleations and propagations. The dislocation mean free path coincides with the characteristic scale of the system, i.e., the grain size. On the opposite scale range (large grain sizes), the breakdown of the HP law stems from the loss of the characteristic scale that was given by the grain size, which restores the marginally stable state characterized by a scale-free distribution of strain bursts.

The HP law thus appears as the signature of an intermediate stage in the polycrystal mechanical response, covering a wide size range between the scale-free critical behavior of single crystals and the specific features of nanograined materials involving individual dislocation processes at a well-defined scale.
The authors are grateful to Professor F. Chmelik for helpful comments on the manuscript.

*Corresponding author.

Email address: louchet@lgge.obs.ujf-grenoble.fr

†Email address: weiss@1gge.obs.ujf-grenoble.fr

${ }^{\ddagger}$ Email address: richeton@lgge.obs.ujf-grenoble.fr

[1] E. O. Hall, Proc. Phys. Soc. London Sect. B 64, 747 (1951).

[2] N. J. Petch, J. Iron Steel Inst., London 173, 25 (1953).

[3] A. Lasalmonie and J.-L. Strudel, J. Mater. Sci. 21, 1837 (1986).

[4] G. Saada, Mater. Sci. Eng. A 400-401, 146 (2005).

[5] U.F. Kocks and H. Mecking, Prog. Mater. Sci. 48, 171 (2003).

[6] G. I. Taylor, J. Inst. Met. 62, 307 (1938).

[7] M.-C. Miguel, A. Vespignani, S. Zapperi, J. Weiss, and J.-R. Grasso, Nature (London) 410, 667 (2001).

[8] M. Koslowski, R. Le Sar, and R. Thomson, Phys. Rev. Lett. 93, 125502 (2004).

[9] T. Richeton, P. Dobron, F. Chmelik, J. Weiss, and F. Louchet, Mater. Sci. Eng. A 424, 190 (2006).

[10] T. Richeton, J. Weiss, and F. Louchet, Nat. Mater. 4, 465 (2005).

[11] J. Friedel, Dislocations (Pergamon, New York, 1964).

[12] T. Richeton, J. Weiss, and F. Louchet, Acta Mater. 53, 4463 (2005).

[13] D. G. Morris, in Mechanical Behaviour of Nanostructured Materials, edited by M. Magini and F. Wohlbier, Materials Science Foundations 2 (Trans Tech Publications, Zurich, 1998).

[14] F. Louchet, in Proceedings of the Seventh International Symposium on Plasticity and Its Current Applications, Cancun (Mexico), 1999, edited by A.S. Khan (Neat Press, Fulton, Maryland, 1999).

[15] F. Louchet and H. Kung, J. of Metastable and Nanocrystalline Materials 7, 55 (2000).

[16] J.C. Li, Transactions of the Society of Petroleum Engineers of the American Institute of Mining, Metallurgical, and Petroleum Engineers, Inc 227, 239 (1963).

[17] P. M. Derlet and H. Van Swygenhoven, Scr. Mater. 47, 719 (2002).

[18] M. Zaiser and P. Moretti, J. Stat. Mech. (2005) P08004.

[19] B. Mintz, Mater. Sci. Technol. 16, 1282 (2000).

[20] M. J. Whelan, P. B. Hirsch, R. W. Horne, and W. Bollmann, Proc. R. Soc. A 240, 524 (1957).

[21] L. E. Murr, Mater. Sci. Eng. 51, 71 (1981).

[22] D. G. Morris, Mechanical Behaviour of Nanostructured Materials (Trans Tech Publications, Zurich, 1998).

[23] G. McMahon and U. Erb, Microstructural Science 17, 447 (1989).

[24] R. J. Hellmig, T. T. Lamark, M. V. Popov, M. Janecek, Y. Estrin, and F. Chmelik, Proc. ISPMA 10 (to be published).

[25] M. Janecek, F. Louchet, B. Doisneau-Cottignies, Y. Bréchet, and N. Guelton, Philos. Mag. A 80, 1605 (2000).

[26] J. S. C. Jang and C. C. Koch, Scr. Metall. Mater. 24, 1599 (1990).

[27] V. Yamakov, D. Wolf, S. R. Phillpot, and H. Gleiter, Acta Mater. 50, 61 (2002). 